\title{
电活性镍钴双金属氧化物高选择性去除/回收水中磷酸盐离子
}

$$
\text { 杨言言 }{ }^{1,2} \text {, 李永国 }{ }^{3} \text {, 祝小雯 }{ }^{1} \text {, 杜 晓 }{ }^{2} \text {, 马旭莉 }{ }^{2} \text {, 郝晓刚 }{ }^{2}
$$

(1. 上饶师范学院 化学与环境科学学院, 上饶 334001; 2. 太原理工大学 化学化工学院, 太原 $030024 ; 3$. 中国辐 射防护研究院, 太原 030006)

摘 要: 磷是植物体生长的重要营养素, 也是引发水体富营养化的重要因素, 因此废水中磷酸盐的去除与回收均至 关重要。本研究采用单极脉冲电沉积法在炭布上制备镍钴双氢氧化物, 并于管式炉中原位焙烧制得镍钴双金属氧化 物(NiCo-Layered Double Oxide, NiCo-LDO)，将其用于电控离子交换(Electrochemically Switched Ion Exchange, ESIX) 过程实现 $\mathrm{PO}_{4}{ }^{3-}$ 的去除与回收。实验对比了 ESIX 与离子交换(Ion Exchange, IX)过程中 NiCo-LDO 对 $\mathrm{PO}_{4}{ }^{3-}$ 的去除性 能, 并考察了其选择性及循环稳定性。结果表明, 在 $(10.00 \pm 0.05) \mathrm{mg} / \mathrm{L}$ 的 $\mathrm{PO}_{4}{ }^{3-}$ 溶液中, ESIX 过程中膜对 $\mathrm{PO}_{4}{ }^{3-}$ 的离 子交换量约为 IX 的 2 倍; NiCo-LDO 对 $\mathrm{PO}_{4}{ }^{3-}$ 具有高选择性，且经过 5 次循环后，离子交换量仍可达到初始值的 $92 \%$ 以上; 结合 XPS 分析, 发现 NiCo-LDO 对 $\mathrm{PO}_{4}{ }^{3-}$ 的 ESIX 过程包括一个不可逆的“记忆效应”结构恢复过程及两个可 逆的层板金属离子氧化/还原和 $\mathrm{PO}_{4}{ }^{3-}$ 与 $\mathrm{O}-\mathrm{H}$ 基团的配体交换过程。

关＼cjkstart键 词：镍钴双金属氧化物; 磷酸盐; 电控离子交换; 配体交换

中图分类号: TQ150 文献标识码: A

\section{Potential Induced Reversible Removal/Recovery of Phosphate Anions with High Selectivity Using an Electroactive NiCo-layered Double Oxide Film}

\author{
YANG Yanyan $^{1,2}$, LI Yongguo ${ }^{3}$, ZHU Xiaowen ${ }^{1}$, DU Xiao ${ }^{2}$, MA Xuli ${ }^{2}$, HAO Xiaogang ${ }^{2}$
}

(1. School of Chemistry and Environmental Science, Shangrao Normal University, Shangrao 334001, China; 2. Department of Chemical Engineering, Taiyuan University of Technology, Taiyuan 030024, China; 3. China Institute for Radiation Protection, Taiyuan 030006, China)

Abstract: Phosphorus is an essential nutrient for organisms growth and a major cause of eutrophication in water bodies. Thus, it is crucial for both of the removal and recovery of phosphate from wastewater. In this work, the NiCo-layered double oxide (NiCo-LDO) was successfully fabricated on carbon cloth conductive substrate via the in-situ calcination of the NiCo-layered double hydroxide (NiCo-LDH) and served as the electrochemically switched ion exchange (ESIX) film electrode for the removal and recovery of $\mathrm{PO}_{4}{ }^{3-}$. The performance of NiCo-LDO for $\mathrm{PO}_{4}{ }^{3-}$ removal by ESIX and ion exchange (IX) was compared, while the selectivity and stability of NiCo-LDO for $\mathrm{PO}_{4}{ }^{3-}$ removal were also investigated. The results revealed that, in $(10.00 \pm 0.05) \mathrm{mg} / \mathrm{L} \mathrm{PO}_{4}{ }^{3-}$ solution, the ion exchange quantity of the NiCo-LDO for $\mathrm{PO}_{4}{ }^{3-}$ removal by ESIX process was about twice over that by IX. Moreover, compared

收稿日期: 2020-06-20; 收到修改稿日期：2020-08-27; 网络出版日期：2020-09-09

基金项目: 国家自然科学基金(21776191，21706181); 上饶师范学院校级自选课题(202028); 山西省重点研发计划 (201803D421094); 国家重点研发计划政府间国际科技创新合作重点专项项目(2017YFE0129200)

National Natural Science Foundation of China (21776191, 21706181); Project of Shangrao Normal University (202028);

Key R\&D project of Shanxi Province (201803D421094); National Key R\&D Program of China (2017YFE0129200)

作者简介: 杨言言(1983-), 女，博士，讲师. E-mail: yangyy0927@163.com

YANG Yanyan (1983-), female, PhD, lecturer. E-mail: yangyy0927@163.com

通信作者: 郝晓刚, 教授.E-mail: xghao@tyut.edu.cn; tyutxghao@hotmail.com HAO Xiaogang, professor. E-mail: xghao@tyut.edu.cn; tyutxghao@hotmail.com 
with $\mathrm{Cl}^{-}, \mathrm{NO}_{3}{ }^{-}, \mathrm{SO}_{4}{ }^{2-}$ and $\mathrm{I}^{-}$, the NiCo-LDO exhibited much higher selectivity towards $\mathrm{PO}_{4}{ }^{3-}$. In addition, the ion exchange quantity still retained $92 \%$ of its initial value after 5 uptake and release cycles. Coupled with XPS analysis, it was found that ESIX process of NiCo-LDO film electrode for $\mathrm{PO}_{4}{ }^{3-}$ removal and recovery mainly consisted of 3 steps, which were an irreversible "memory effect" structure recovery process, the redox reaction of metal ion in lamellar and the ligand exchange between $\mathrm{PO}_{4}{ }^{3-}$ and $\mathrm{O}-\mathrm{H}$ groups.

Key words: NiCo-layered double oxide; phosphate; electrochemically switched ion exchange; ligand exchange

近年来，水体中由人类活动排放的各类污染物， 如放射性核素 ${ }^{[1]}$ 、重金属 ${ }^{[2] 、}$ 、阴离子 ${ }^{[3]}$ 、有机染料 ${ }^{[4]}$ 等, 对人类健康及环境生态系统造成严重威胁。磷 酸盐作为生物体生长的重要营养素 ${ }^{[3]}$, 其含量过高 会造成水体“富营养化”[5-6], 引发水生植被疯长、消 耗水中溶解氧进而引起水中好氧生物死亡。因此, 开发行之有效的除磷/回收磷技术, 对于环境保护及 废物资源化利用均具有重要意义。常用除磷技术如 化学沉淀法、强化生物除磷等, 常面临出口磷浓度 偏高等严峻挑战 ${ }^{[7]}$ 。选择性吸附可实现出口磷浓度 低, 且有可能回收高纯度的磷酸盐, 因此被认为是 一种直接有效的深度除磷技术 ${ }^{[8]}$ 。但是与其他共存 离子相比, $\mathrm{PO}_{4}{ }^{3-}$ 具有较高的水合能且其浓度普遍较 低, 致使常用的吸附剂除磷效果不佳 ${ }^{[9]}$ 。另外, 使用 强碱再生吸附剂还会带来一定的二次污染 ${ }^{[10]}$ 。

电控离子交换(Electrically Switched Ion Exchange, ESIX) 是电化学与离子交换相结合的离子选择性分 离技术 ${ }^{[11]}$, 通过调节具有特定离子识别能力的电活 性离子交换膜的氧化/还原电位来控制离子的置入/ 释放。因此, ESIX 可快速分离溶液中极低浓度的离 子, 且离子交换膜无需化学再生, 可最大限度地消 除二次污染，已被用于 $\mathrm{Cs}^{+[12]} 、 \mathrm{Ba}^{2+[13]} 、 \mathrm{Ni}^{2+[14]}$ 、 $\mathrm{Pb}^{2+[15]} 、 \mathrm{Y}^{3+[16]}$ 等阳离子及 $\mathrm{ClO}_{4}^{-[17]} 、 \mathrm{~F}^{-[18]} 、 \mathrm{I}^{-[12]}$ 等 阴离子的选择性分离。

研究发现, 离子最外层电子及空位轨道决定了 离子间的键合能力 ${ }^{[19]}$ 。 $\mathrm{PO}_{4}{ }^{3-}$ 提供孤立电子对的能力 比其他共存离子强，其吸附过程可通过与金属之间 的络合来实现 ${ }^{[7]}$ 。鉴于此, 由不同金属氢氧化物层及 被阴离子和水分子占据插层空间的层状双氢氧化物 (Layered double hydroxides, LDHs) ${ }^{[20]}$ 可用于选择性 除磷。特别是, LDHs 具有独特的二维层状结构、比 表面积大且层板金属离子及层间阴离子可调，有利 于吸附阴离子 ${ }^{[4]}$ 。焙烧 LDHs 可得到双金属氧化物 (Layered double Oxide, LDO), 其层状结构恢复过程 可进一步提高阴离子的去除效率 ${ }^{[10]}$ 。此外, 过渡金 属组成的 LDHs 可以有效调节层板电荷分布, 通常 具有良好的电活性 ${ }^{[21]}$ 。因此, 将 NiCo-LDO 作为电 活性膜应用于 ESIX 过程有望实现 $\mathrm{PO}_{4}{ }^{3-}$ 的高效、选
择性去除与回收。

本工作采用单极脉冲电沉积法(Unipolar pulse electrodeposition, UPED)在炭布上制备 NiCo-LDH, 并在管式炉中原位焙烧制得 NiCo-LDO, 将其用于 ESIX 过程分离/回收水中的 $\mathrm{PO}_{4}{ }^{3-}$; 通过控制电位, 考察 NiCo-LDO 对 $\mathrm{PO}_{4}{ }^{3-}$ 的分离性能; 结合 XPS 分 析，探讨 NiCo-LDO 选择性分离/回收 $\mathrm{PO}_{4}{ }^{3-}$ 的 ESIX 机理，为其工业应用提供理论依据。

\section{1 实验方法}

\section{1 试剂与仪器}

所用试剂均为分析纯或优级纯，且均未经过二 次提纯。溶液均使用超纯水(Millipore $18.2 \mathrm{M} \Omega \cdot \mathrm{cm}$ ) 配制。电化学实验均在 EC-Lab 软件控制的 VMP3 Princeton 电化学工作站上进行, 采用三电极体系, 参比电极为 $\mathrm{Ag} / \mathrm{AgCl}$ 、对电极为铂片 $(5 \mathrm{~cm} \times 2 \mathrm{~cm} \times$ $0.1 \mathrm{~cm}$ )、工作电极为导电基体或膜电极。采用扫描 电子显微镜(SEM, Zeiss SUPRA 55)及透射电子显微 镜(TEM, TECNAI F30, USA)观察膜表面形貌。通过 $X$ 射线光电子能谱(XPS)检测材料元素组成。采用 $X$ 射线衍射(XRD, Rigaku SmartLab, Japan)测定膜的 晶体结构。采用离子色谱仪(DX-600, DIONEX, USA) 测定溶液中阴离子浓度。

\section{$1.2 \mathrm{NiCo}-\mathrm{LDO}$ 的制备}

基体预处理：炭布 $(2 \mathrm{~cm} \times 5 \mathrm{~cm} \times 0.1 \mathrm{~cm})$, 用无 水乙醇浸泡 2 3 h 以除去表面有机物。

NiCo-LDO 的制备: 采用 UPED 在经预处理的 炭布上制备 NiCo-LDH; 取出后用超纯水冲洗干净, 置在 $60{ }^{\circ} \mathrm{C}$ 的真空干燥箱中过夜; 称重后置于管式 炉中在 $300{ }^{\circ} \mathrm{C}$ 下恒温焙烧 $2 \mathrm{~h}$, 即得 NiCo-LDO。其 中, 制备液: $0.09 \mathrm{~mol} / \mathrm{L} \mathrm{Ni}\left(\mathrm{NO}_{3}\right)_{2}$ 与 $0.01 \mathrm{~mol} / \mathrm{L}$ $\mathrm{Co}\left(\mathrm{NO}_{3}\right)_{2}$; 制备参数: 脉冲电压, $-1.0 \mathrm{~V}$; 开、关时间, $1.0 \mathrm{~s}$; 脉冲次数, 500 次。本工作中所提到的电位均 相对于 $\mathrm{Ag} / \mathrm{AgCl}$ 参比电极。

\section{3 电化学性能实验}

在 $200 \mathrm{~mL}$ 的 $\mathrm{Na}_{3} \mathrm{PO}_{4}$ 溶液中, 采用恒电位法检 
测 NiCo-LDO 对 $\mathrm{PO}_{4}{ }^{3-}$ 的吸/脱附性能。首先对比 ESIX 及 IX 技术对 $\mathrm{PO}_{4}{ }^{3-}((10.00 \pm 0.05) \mathrm{mg} / \mathrm{L})$ 吸附性能的影 响; 然后在含 $\mathrm{PO}_{4}{ }^{3-}(21.19 \mathrm{mg} / \mathrm{L}) 、 \mathrm{SO}_{4}{ }^{2-}(21.71 \mathrm{mg} / \mathrm{L})$ 、 $\mathrm{Cl}^{-}(21.64 \mathrm{mg} / \mathrm{L})$ 和 $\mathrm{I}^{-}(22.85 \mathrm{mg} / \mathrm{L})$ 的四元混合溶液中, 采用竞争吸附的方式考察 NiCo-LDO 对 $\mathrm{PO}_{4}{ }^{3-}$ 的吸 附选择性; 最后测试 NiCo-LDO 对 $\mathrm{PO}_{4}{ }^{3-}((26.00 \pm$ $0.68) \mathrm{mg} / \mathrm{L}$ )的吸附/脱附稳定性。

吸附电位, 即氧化电位为 $0.8 \mathrm{~V}$; 脱附电位, 即 还原电位为 $-0.4 \mathrm{~V}$; 吸/脱附时间均为 $6 \mathrm{~h}$ 。IX 为开 路电压。吸附量、脱附量、脱附率及分离因子分别 采用式(1)、(2)、(3)及(4)计算:

$$
\begin{gathered}
Q_{\mathrm{a}}=\frac{\left(C_{0}-C\right) \times V}{m} \\
Q_{\mathrm{d}}=\frac{\left(C-C_{0}\right) \times V}{m} \\
\text { Desorption ratio }=\frac{Q_{\mathrm{d}}}{Q_{a}} \\
\alpha_{\mathrm{A}^{n-}}^{\mathrm{PO}_{4}^{3-}}=\frac{x_{\mathrm{PO}_{4}{ }^{3-}}^{\prime} / x_{\mathrm{A}^{n-}}^{\prime}}{x_{\mathrm{PO}_{4}{ }^{3-}} / x_{\mathrm{A}^{n-}}}
\end{gathered}
$$

式(1)、(2)及(3)中, $Q_{\mathrm{a}} 、 Q_{\mathrm{d}}$ 为吸附量、脱附量 $(\mathrm{mg} / \mathrm{g})$, $C_{0} 、 C$ 为初始和各检测点 $\mathrm{PO}_{4}{ }^{3-}$ 的浓度 $(\mathrm{mg} / \mathrm{L}), V$ 是 溶液体积 $(\mathrm{L}), m$ 是 NiCo-LDO 的质量 $(\mathrm{g})$; 式(4)中, 分子为膜内阴离子摩尔分数比值, 分母为溶液中初 始阴离子摩尔分数比值, $\mathrm{A}^{n-}$ 指 $\mathrm{SO}_{4}{ }^{2-} 、 \mathrm{Cl}^{-}$或 $\mathrm{I}^{-}$。

将制备好的 NiCo-LDO 膜在 $0.1 \mathrm{~mol} / \mathrm{L} \mathrm{Na} \mathrm{Na}_{3} \mathrm{PO}_{4}$ 溶液中设定电位为 $0.8 \mathrm{~V}$ 氧化 $10 \mathrm{~min}$, 在控制电位 下取出冲洗、晾干; 通过 XPS 检测氧化后 NiCo-LDO 膜的化学组成及元素价态。

\section{2 结果与讨论}

\subsection{NiCo-LDO 的表征}

图 1 为 $\mathrm{NiCo}-\mathrm{LDH}$ 焙烧前后的 SEM 照片及 NiCo-LDO 的 TEM 照片。从 SEM 照片中可以发现, 焙烧前后的样品均呈现典型的纳米片层状结构, 与 NiCo-LDH 相比, NiCo-LDO 的纳米片尺寸更加均 一、形貌也更加平整, 纳米片之间的孔隙也更大且 更加均匀, 说明其排列方式更加疏松有序。这种孔 隙均匀的多孔蜂窝状结构, 将更加有助于电荷与离 子的传输, 从而使膜的电活性更高 ${ }^{[22]}$ 。

从 NiCo-LDO 的 TEM 照片(图 1(e))可以发现, 其呈现出明显的球形纳米颗粒结构。此外, 根据高 分辨率透射电镜照片(图 1(f)), 可观察到晶格间距条 纹, 且间距约为 $0.20 \mathrm{~nm}$, 对应于 $\mathrm{NiO}$ 的(200) 晶面 ${ }^{[23]}$ 。
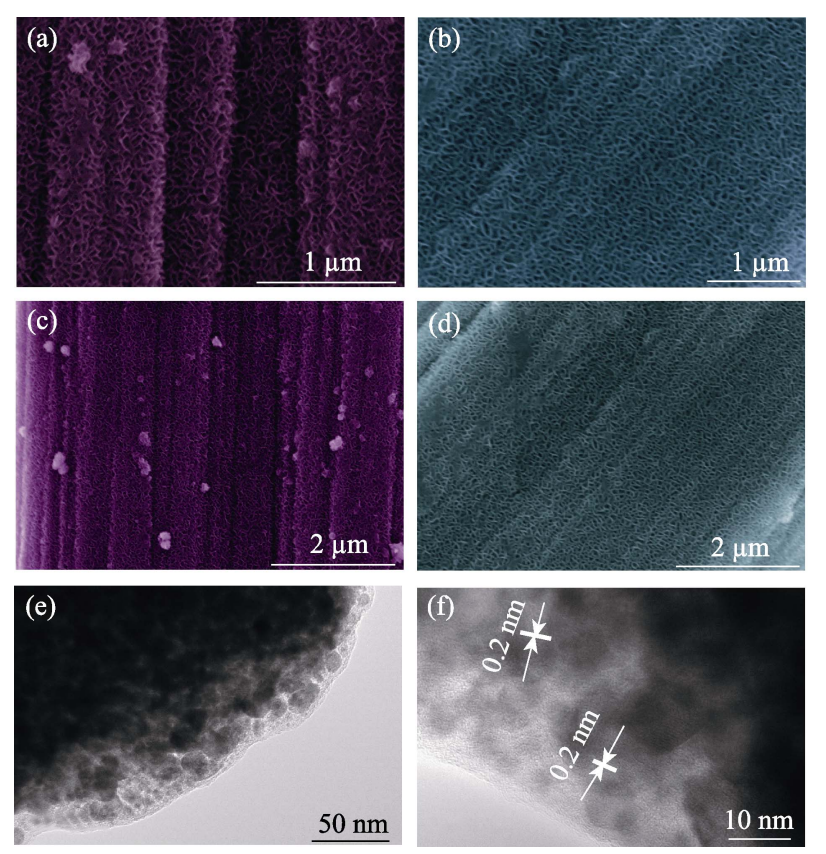

图 1 NiCo-LDH (a, c) 和 NiCo-LDO (b, d) 的 SEM 照片及 NiCo-LDO 的 TEM 照片(e)和 HRTEM 照片(f)

Fig. 1 SEM images of NiCo-LDH (a, c) and NiCo-LDO (b, d), TEM (e) and HRTEM (f) images of NiCo-LDO

采用 XRD 及 XPS 分析了 NiCo-LDO 的晶体结 构及表面元素组成。图 2(a)为 NiCo-LDH 焙烧前后 的 XRD 图谱, 仅在 $2 \theta=26^{\circ} 、 54^{\circ}$ 左右观察到两个比 较尖锐的峰, 与碳基体的特征峰对应, 这是由于 NiCo-LDH 和 NiCo-LDO 沉积量少造成的。从局部 放大的 LDH 图谱中可观察到 LDHs 的(003)、(012)、 (018)及(110)典型特征峰。而 LDO 的图谱中, $\operatorname{LDHs}(003)$ 峰消失, 同时出现新的典型金属氧化物的峰, 分别

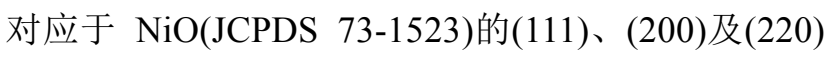
晶面的衍射峰 ${ }^{[23]}$, 这也证实经过焙烧, $\mathrm{NiCo}-\mathrm{LDH}$ 转 变为 $\mathrm{NiO}$ 晶型的 $\mathrm{NiCo}-\mathrm{LDO}$ 。从 XPS 全谱图(图 2(b)) 中也可以观察到 NiCo-LDO 及炭布基体的组成元素 $\mathrm{Co} 、 \mathrm{Ni} 、 \mathrm{O}$ 和 $\mathrm{C}$ 的峰。

另外, $\mathrm{Co} 2 \mathrm{p}$ 高分辨率光谱(图 2(c)) 可与两个自 旋轨道偶极子相匹配。结合能约为 781.8 和 $797.3 \mathrm{eV}$ 的两个峰对应于 $\mathrm{Co}^{2+}$, 而结合能在 780.3 和 $795.8 \mathrm{eV}$ 的峰分别对应 $\mathrm{Co}^{3+[24]}$ 。 Ni2 $\mathrm{p}$ 的高分辨率光谱中(图 2(d)), 在 874.1 和 $856.3 \mathrm{eV}$ 处的两个自旋轨道对分别对应 于 $\mathrm{Ni}^{3+}$ 的 $N i 2 p_{1 / 2}$ 和 $N i 2 p_{3 / 2}$ 的特征峰, 而在 872.4 和 $854.7 \mathrm{eV}$ 处的两个自旋轨道对则分别对应于 $\mathrm{Ni}^{2+}$ 的 $\mathrm{Ni} 2 \mathrm{p}_{1 / 2}$ 和 $\mathrm{Ni} 2 \mathrm{p}_{3 / 2}$ 的特征峰 ${ }^{[25]}$ 。

\section{2 电化学吸附 $\mathrm{PO}_{4}{ }^{3-}$}

为了考察 $\mathrm{NiCo}-\mathrm{LDO}$ 电化学分离 $\mathrm{PO}_{4}{ }^{3-}$ 的实际 效率, 图 3(a)给出了采用不同分离技术时, NiCo-LDO 对 $\mathrm{PO}_{4}{ }^{3-}$ 的吸附动力学曲线。与 IX 相比, ESIX 过程 

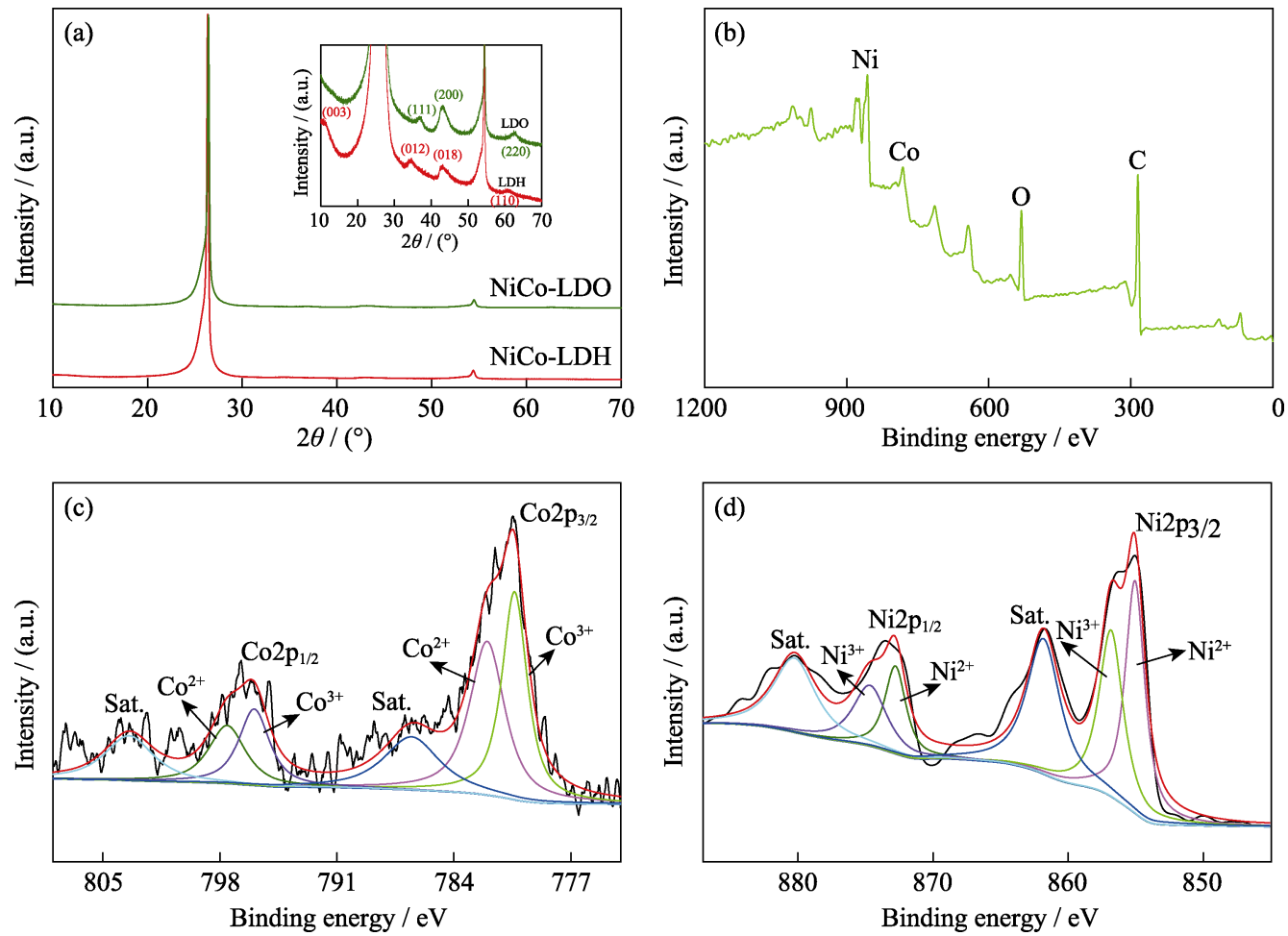

图 2 NiCo-LDH 焙烧前后的 XRD 图谱(a)、NiCo-LDO 的 XPS 全谱(b)、Co2p(c)及 $\mathrm{Ni2p}(\mathrm{d})$ 高分辨率 XPS 谱

Fig. 2 XRD patterns of NiCo-LDH and NiCo-LDO (a), full XPS (b), high resolution Co2p (c), and Ni2p (d) spectra of NiCo-LDO
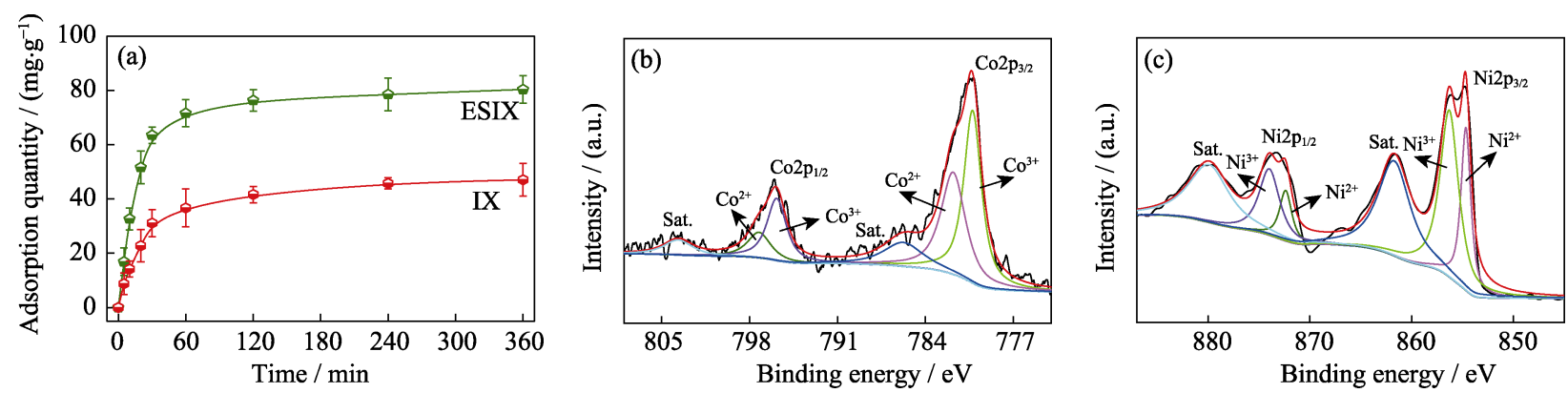

图 3 NiCo-LDO 在 ESIX 及 IX 过程中对 $\mathrm{PO}_{4}{ }^{3-}$ 的吸附曲线( $\mathrm{a}$ 、、 NiCo-LDO 氧化后 $\mathrm{Co} 2 \mathrm{p}(\mathrm{b})$ 及 $\mathrm{Ni} 2 \mathrm{p}(\mathrm{c})$ 高分辨率 XPS 谱

Fig. 3 Kinetic adsorption curves of $\mathrm{PO}_{4}{ }^{3-}$ on NiCo-LDO under ESIX or IX process (a), high resolution Co2p (b) and Ni2p (c) spectra of NiCo-LDO after oxidation

中 NiCo-LDO 对 $\mathrm{PO}_{4}{ }^{3-}$ 的吸附量明显更高。很显然, 通过施加氧化电位可以显著提高吸附效率, ESIX 过 程的饱和吸附量 $(80.36 \mathrm{mg} / \mathrm{g})$ 约为 IX $(47.10 \mathrm{mg} / \mathrm{g})$ 的 2 倍。此外, 在到达吸附平衡前, ESIX 过程的吸附速 率也远高于 IX, 这可归因于以下两个方面: (i)离子 扩散推动力: ESIX 过程包括电势梯度和浓度梯度, 而 IX 仅有浓度梯度 ${ }^{[26]}$; (ii)层板正电荷数：ESIX 氧 化过程, 层板正电荷数增加(对比图 3(b)和图 2(c)以 及图 3(c)和图 2(d)), 这有利于层板通过静电吸引结 合 $\mathrm{PO}_{4}{ }^{3-}$, 而 IX 则不存在这一过程。

\section{3 选择性分析}

图 4 为 $\mathrm{NiCo}-\mathrm{LDO}$ 对 $\mathrm{PO}_{4}{ }^{3-} 、 \mathrm{SO}_{4}{ }^{2-} 、 \mathrm{I}^{-}$和 $\mathrm{Cl}^{-}$的 竞争吸附动力学曲线, 计算得到 $\mathrm{PO}_{4}{ }^{3-} / \mathrm{SO}_{4}{ }^{2-} 、 \mathrm{PO}_{4}{ }^{3-} / \mathrm{I}^{-}$

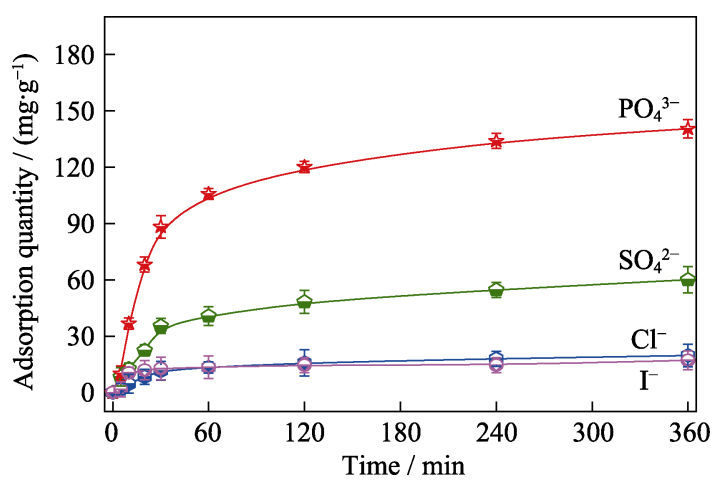

图 $4 \mathrm{NiCo}-\mathrm{LDO}$ 在四元混合溶液中对 $\mathrm{PO}_{4}{ }^{3-} 、 \mathrm{SO}_{4}{ }^{2-} 、 \mathrm{I}^{-}$和 $\mathrm{Cl}^{-}$的竞争吸附曲线

Fig. 4 Competitive adsorption kinetics curves of $\mathrm{PO}_{4}{ }^{3-}, \mathrm{SO}_{4}{ }^{2-}$, $\mathrm{I}^{-}$, and $\mathrm{Cl}^{-}$onto NiCo-LDO in $21.19 \mathrm{mg} / \mathrm{L} \mathrm{PO}_{4}{ }^{3-}, 21.71 \mathrm{mg} / \mathrm{L}$ $\mathrm{SO}_{4}{ }^{2-}, 21.64 \mathrm{mg} / \mathrm{L} \mathrm{I}^{-}$, and $22.85 \mathrm{mg} / \mathrm{L} \mathrm{Cl}^{-}$mixed solution 
表 1 NiCo-LDH 与阴离子之间结合能计算结果

Table 1 Calculated binding energy between anion-and NiCo-LDH

\begin{tabular}{lc}
\hline Anion-LDH & $E_{\mathrm{cp}} /\left(\mathrm{kJ} \cdot \mathrm{mol}^{-1}\right)$ \\
\hline $\mathrm{Cl}^{-}-\mathrm{LDH}$ & -455.88 \\
$\mathrm{I}^{-}-\mathrm{LDH}$ & -345.20 \\
$\mathrm{SO}_{4}{ }^{2-}-\mathrm{LDH}$ & -813.90 \\
$\mathrm{PO}_{4}{ }^{3-}-\mathrm{LDH}$ & -868.36 \\
\hline
\end{tabular}

*These calculated results do not take into account the effect of aqueous solution and are only used as an auxiliary explanation for the selective results

和 $\mathrm{PO}_{4}{ }^{3-} / \mathrm{Cl}^{-}$分离因子分别为 2.34、8.16 和 7.12, 这 说明相对于 $\mathrm{SO}_{4}{ }^{2-} 、 \mathrm{I}^{-}$和 $\mathrm{Cl}^{-}$来说, $\mathrm{NiCo}-\mathrm{LDO}$ 对 $\mathrm{PO}_{4}{ }^{3-}$ 表现出更优的选择性。采用 Accelrys Material Studio 6.0 程序包中 DMOL3 模块的密度泛函理论计算分 别得到 $\mathrm{PO}_{4}{ }^{3-} 、 \mathrm{SO}_{4}{ }^{2-} 、 \mathrm{I}^{-}$和 $\mathrm{Cl}^{-}$与 $\mathrm{NiCo}-\mathrm{LDH}$ 的结合 能数据, 结果如表 1 所示, $\mathrm{NiCo}-\mathrm{LDH}$ 与 $\mathrm{PO}_{4}{ }^{3-}$ 的结 合能明显低于与其他阴离子的结合能，从侧面说明 在相同条件下, NiCo-LDO 会优先结合 $\mathrm{PO}_{4}{ }^{3-}$ 。另外 比较容易理解的是, 带有较多负电荷数的阴离子更 容易被带正电荷的 LDHs 吸附 ${ }^{[27]}$ 。

\section{4 稳定性实验}

在初始浓度为 $(26.00 \pm 0.68) \mathrm{mg} / \mathrm{L}$ 的 $\mathrm{Na}_{3} \mathrm{PO}_{4}$ 溶液 中测试 NiCo-LDO 的再生性及循环稳定性。如图 5 所示, 在每一次吸/脱附循环中, 脱附率均可达到 $92 \%$ 以上，这说明通过调节 NiCo-LDO 的氧化还原 状态可以很好地实现 $\mathrm{PO}_{4}{ }^{3-}$ 的吸附与脱附。第一循环 的脱附率相对低, 可能是由于部分被吸附的 $\mathrm{PO}_{4}{ }^{3-}$ 作为层间阴离子所致 ${ }^{[17]}$ 。此时 $\mathrm{NiCo}-\mathrm{LDO}$ 也转化为 $\mathrm{NiCo}-\mathrm{LDH}$, 这可由其后四个循环中的吸附量与脱 附量均与第一循环的脱附量相差无几推测得出。此 后, 随着循环次数的增加, $\mathrm{NiCo}-\mathrm{LDO} / \mathrm{NiCo}-\mathrm{LDH}$ 的 离子交换量保持稳定。其中, 第一循环为 $159.36 \mathrm{mg} / \mathrm{g}$, 第二、三、四、五循环均在 $146 \mathrm{mg} / \mathrm{g}$ 以上，与表 2 中所列其他吸附剂的吸附量相比也相对较高。随循

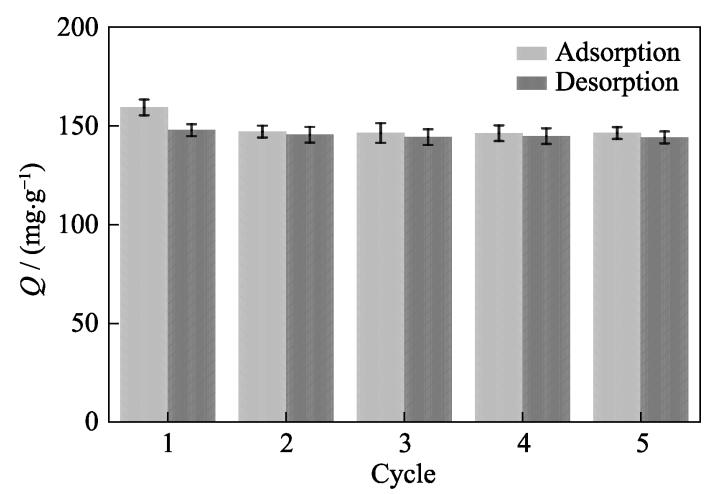

图 $5 \mathrm{NiCo-LDO}$ 的稳定性测试结果

Fig. 5 Recyclability test of NiCo-LDO for the removal of $\mathrm{PO}_{4}{ }^{3-}$
表 2 文献报道的 $\mathrm{PO}_{4}{ }^{3-}$ 吸附剂对 $\mathrm{PO}_{4}{ }^{3-}$ 吸附量的对比

Table 2 Comparison of the ion adsorption quantity with those reported $\mathrm{PO}_{4}{ }^{3-}$ ion adsorbents

\begin{tabular}{lcrc}
\hline \multicolumn{1}{c}{ Ion adsorbent } & $\begin{array}{c}\text { Adsorption } \\
\text { quantity } /\left(\mathrm{mg}^{-1} \mathrm{~g}^{-1}\right)\end{array}$ & Time/h & Ref. \\
\hline Aluminum oxide hydroxide & 36.27 & 4 & {$[6]$} \\
Fe-Mn binary oxide & 33.2 & 24 & {$[29]$} \\
Hydroxy-aluminum & 12.7 & 7 & {$[30]$} \\
MgFe-Zr-LDH@magnetic & 30 & 24 & {$[31]$} \\
particles & 67.29 & 8 & {$[32]$} \\
Am-ZrO & & 6 & {$[10]$} \\
$\mathrm{Mg}-\mathrm{Fe}-\mathrm{Cl} \mathrm{LDH}$ & 9.8 & 10 & {$[33]$} \\
$\mathrm{Nano}-\mathrm{La}(\mathrm{III})($ hydr)oxides & $\sim 55$ & 5 & {$[5]$} \\
$\mathrm{La}(\mathrm{OH})_{3} / \mathrm{Fe}{ }_{3} \mathrm{O}_{4}$ & 83.5 & 6 & This work \\
$\mathrm{NiCo}-\mathrm{LDO}$ & 159.36 & & \\
\hline
\end{tabular}

环次数增加, 吸附量未出现明显降低, 这与膜疏松 有序的纳米结构有关。上述结果表明, NiCo-LDO 是 一种具有高吸附量、良好选择性及循环稳定性的分 离与回收 $\mathrm{PO}_{4}{ }^{3-}$ 的 ESIX 材料。

\subsection{ESIX 机理分析}

图 6 所示的是 NiCo-LDO 氧化前后的 P2p 及 $\mathrm{O} 1 \mathrm{~s}$ 的高分辨率 XPS 图谱。从图 6(A)中可以发现，氧化 前 $\mathrm{P} 2 \mathrm{p}$ 图谱未检测到 $\mathrm{P}$ 元素的存在，而氧化后的图 谱中出现一个很明显的 $\mathrm{P}$ 元素峰, 证实了通过电化 学氧化, $\mathrm{PO}_{4}{ }^{3-}$ 可以被置入膜内。如图 6(B) 所示, $\mathrm{O} 1 \mathrm{~s}$ 图谱可被分解成三个在 530.0、531.4 和 $532.3 \mathrm{eV}$ 处 的高斯峰, 分别对应于 $\mathrm{M}-\mathrm{O}, \mathrm{O}-\mathrm{H}$ 基团和水中的氧 原子 ${ }^{[28]}$ 。氧化前, NiCo-LDO 中仅含有非常少量的水 分子, 说明层间大部分水分子在炦烧中失去; 氧化 过程吸附 $\mathrm{PO}_{4}{ }^{3-}$ 后，水分子的含量也明显增大，说明 此时 LDO 通过“记忆效应”恢复了 LDH 结构，水分 子与 $\mathrm{PO}_{4}{ }^{3-}$ 同时进入 $\mathrm{LDH}$ 的插层空间。另外, 氧化 后 $\mathrm{M}-\mathrm{O}$ 基团相对含量增大, $\mathrm{O}-\mathrm{H}$ 基团相对含量减小, 说明 $\mathrm{NiCo}-\mathrm{LDO}$ 对 $\mathrm{PO}_{4}{ }^{3-}$ 的吸附可能还存在 $\mathrm{PO}_{4}{ }^{3-}$ 与 层板金属离子形成络合物，同时释放 $\mathrm{O}-\mathrm{H}$ 的配体交换 过程 ${ }^{[27-28]}$ 。再对比图 3(b)和图 2(c) 以及图 3(c) 和图 2(d), 氧化后层板三价金属离子增多, 即层板所带正电荷 数增多, 这有利于增强静电对 $\mathrm{PO}_{4}{ }^{3-}$ 的吸附。

综上所述, ESIX 的氧化过程, 即 $\mathrm{PO}_{4}{ }^{3-}$ 的去除过 程(如图 7 所示)应包括以下三个分过程: (i)LDO 的 “记忆效应”, 通过吸附水中的 $\mathrm{PO}_{4}{ }^{3-}$ 及水分子以恢 复 LDH 结构; (ii)层板金属离子的电化学氧化作用, 进一步增强层板对 $\mathrm{PO}_{4}{ }^{3-}$ 的静电吸引; (iii) $\mathrm{PO}_{4}{ }^{3-}$ 与 $\mathrm{O}-\mathrm{H}$ 之间的配体交换过程。另外，结合稳定性实验 中第一循环脱附量低的结果，可知 LDO 的“记忆效 应”为不可逆过程。因此, ESIX 的还原过程, 即 $\mathrm{PO}_{4}{ }^{3-}$ 的回收过程，则仅包括两个分过程，即层板金属离 子被还原, 层板带电荷数减少, 静电吸附的 $\mathrm{PO}_{4}{ }^{3}$ 被 释放; 配体交换的 $\mathrm{PO}_{4}{ }^{3-}$ 也会重新被 $\mathrm{O}-\mathrm{H}$ 取代。 

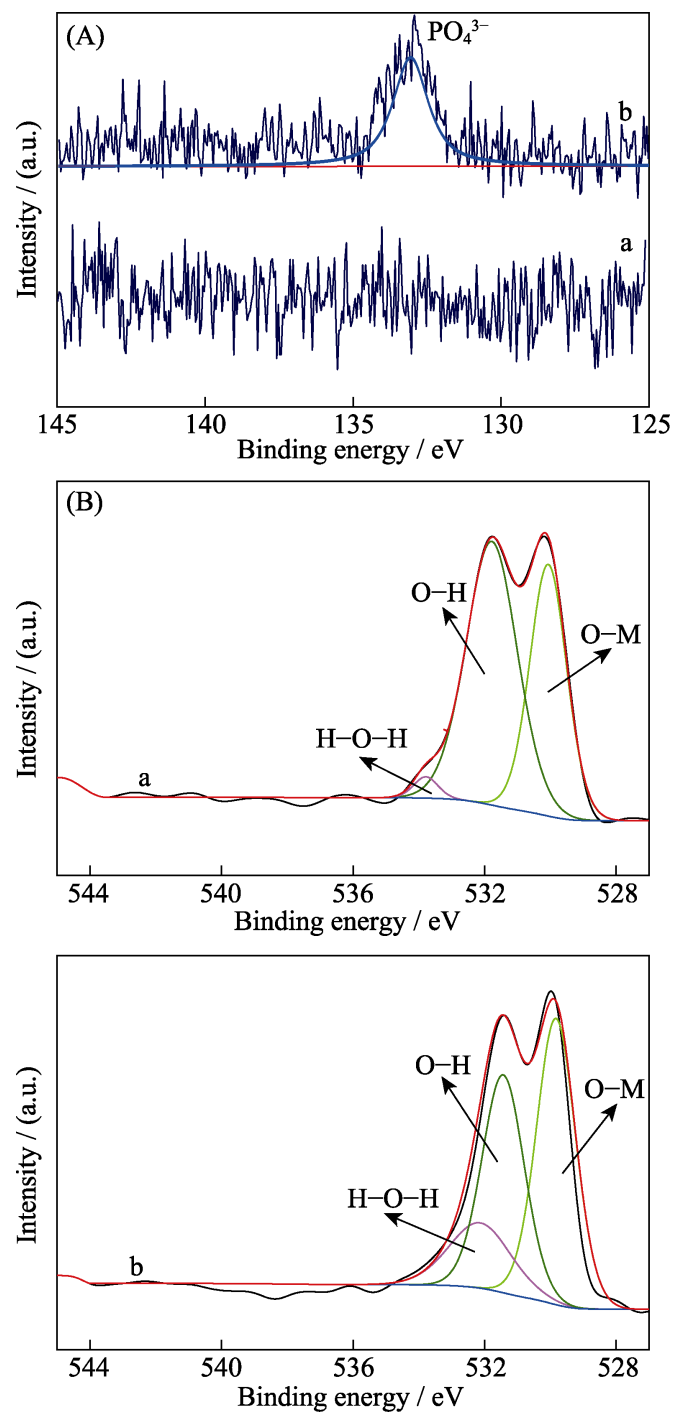

图 6 NiCo-LDO 氧化前(曲线组 a)后(曲线组 b)P2p (A)及 $\mathrm{O} 1 \mathrm{~s}(\mathrm{~B})$ 的高分辨率 XPS 图谱

Fig. 6 High resolution P2p (A) and O1s (B) spectra of NiCo-LDO before (curves a) and after (curves b) oxidation

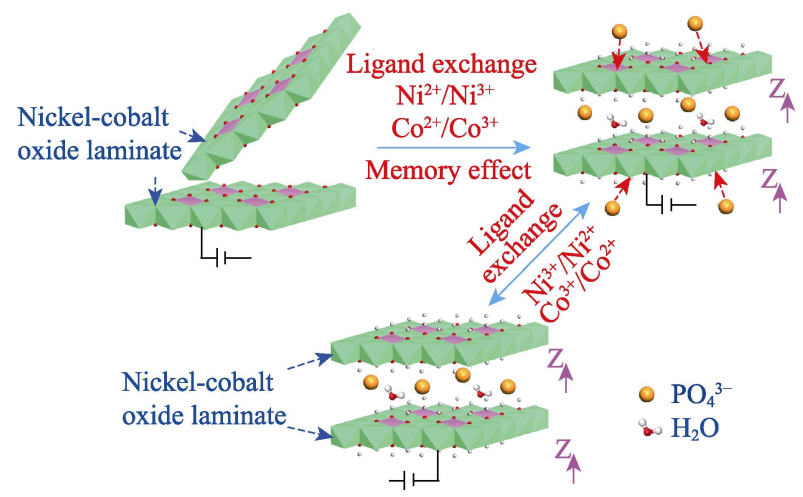

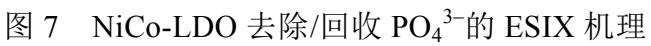

Fig. 7 Illustration of the ESIX mechanism of NiCo-LDO for removal/recovery of $\mathrm{PO}_{4}{ }^{3-}$

\section{3 结论}

采用 UPED 在炭布上制备 NiCo-LDH，在管式炉
中焙烧制得 NiCo-LDO，并通过 XRD、SEM、TEM 及 XPS 等手段表征。主要结论如下:

1)与 IX 相比, ESIX 过程具有更快的 $\mathrm{PO}_{4}{ }^{3-}$ 吸附速 率及更高的 $\mathrm{PO}_{4}{ }^{3}$ 吸附量, 其饱和吸附量约为 $\mathrm{IX}$ 的 2 倍;

2) NiCo-LDO 对 $\mathrm{PO}_{4}{ }^{3-}$ 具有高的选择性, 且具有 良好的再生性及循环稳定性, 经过 5 次循环后, 吸 附量仍可达初始值的 $92 \%$ 以上;

2)结合 XPS 分析及稳定性结果表明, NiCo-LDO 分离/回收 $\mathrm{PO}_{4}{ }^{3-}$ 的 ESIX 过程包括一个不可逆的“记 忆效应”结构恢复过程和两个可逆过程，即层板金属 的可逆氧化还原及 $\mathrm{PO}_{4}^{3-}$ 与 $\mathrm{O}-\mathrm{H}$ 之间的可逆配体交换。

\section{参考文献:}

[1] WANG XIANG-XUE, YU SHU-JUN, WANG XIANG-KE. Removal of radionuclides by metal-organic framework-based materials. Journal of Inorganic Materials, 2019, 34(1): 17-26.

[2] WANG XIANG-XUE, LI XING, WANG JIA-QI, et al. Recent advances in carbon nitride-based nanomaterials for the removal of heavy metal ions from aqueous solution. Journal of Inorganic Materials, 2020, 35(3): 260-270.

[3] XIONG W, TONG J, YANG Z, et al. Adsorption of phosphate from aqueous solution using iron-zirconium modified activated carbon nanofiber: performance and mechanism. Journal of Colloid and Interface Science, 2017, 493: 17-23.

[4] ZHANG XIAO-FENG, ZHANG GUAN-HUA, MENG YUE, et al. Photocatalytic degradation of methylene blue by Schiff-base cobalt modified $\mathrm{CoCr}$ layered double hydroxides. Journal of Inorganic Materials, 2019, 34(9): 974-982.

[5] WU B, FANG L, FORTNER J D, et al. Highly efficient and selective phosphate removal from wastewater by magnetically recoverable $\mathrm{La}(\mathrm{OH})_{3} / \mathrm{Fe}_{3} \mathrm{O}_{4}$ nanocomposites. Water Research, 2017, 126: $179-188$.

[6] TANADA S, KABAYAMA M, KAWASAKI N, et al. Removal of phosphate by aluminum oxide hydroxide. Journal of Colloid and Interface Science, 2003, 257(1): 135-140.

[7] WU B, WAN J, ZHANG Y, et al. Selective phosphate removal from water and wastewater using worption: process fundamentals and removal mechanisms. Environmental Science \& Technology, 2020, 54(1): 50-66.

[8] MAYER B, GERRITY D, RITTMANN B, et al. Innovative strategies to achieve low total phosphorus concentrations in high water flows. Critical Reviews in Environmental Science \& Technology, 2013, 43(4): 409-441.

[9] LIU R, CHI L, WANG X, et al. Review of metal(hydr)oxide and other adsorptive materials for phosphate removal from water. Journal of Environmental Chemical Engineering, 2018, 6(4): 5269-5286.

[10] ASHEKUZZAMAN S, JIANG J. Strategic phosphate removal/ recovery by a reusable $\mathrm{Mg}-\mathrm{Fe}-\mathrm{Cl}$ layered double hydroxide. Process Safety and Environmental Protection, 2017, 107: 454-462.

[11] XIAO JUN-QIANG, HAO XIAO-GANG. Electrochemically switched ion exchange. Progress in Chemistry, 2010, 22(12): 2420-2427.

[12] LIAO S, XUE C, WANG Y, et al. Simultaneous separation of iodide and cesium ions from dilute wastewater based on PPy/PTCF and NiHCF/PTCF electrodes using electrochemically switched ion exchange method. Separation and Purification Technology, 2015, 139: 63-69.

[13] JU JIAN, HAO XIAO-GANG, ZHANG ZHONG-LIN, et al. 
Electrochemically controlled ion separation performances of electrodeposited nickel hexacyanoferrate thin films in alkaline earth metal solution. Journal of Inorganic Materials, 2008, 23(6): $1115-1120$.

[14] DU X, ZHANG H, HAO X, et al. Facile preparation of ionimprinted composite film for selective electrochemical removal of nickel (II) ions. ACS Applied Materials \& Interfaces, 2014, 6(12): 9543-9549.

[15] ZHANG Q, DU X, MA X, et al. Facile preparation of electroactive amorphous $\alpha$-ZrP/PANI hybrid film for potential-triggered adsorption of $\mathrm{Pb}^{2+}$ ions. Journal of Hazardous Materials, 2015, 289: 91-100.

[16] DU X, SUN X, ZHANG H, et al. A facile potential-induced in-situ ion removal trick: fabrication of high-selective ion imprinted film for trivalent yttrium ion separation. Electrochimica Acta, 2015, 176: 1313-1323.

[17] YANG Y, DU X, AN X, et al. Potential-induced reversible uptake/release of perchlorate from wastewater by polypyrrole@ CoNi-layered double hydroxide modified electrode with protonligand effect. Journal of Colloid and Interface Science, 2018, 523: 159-168.

[18] YANG Y, DU X, ABUDULA A, et al. Highly efficient defluoridation using a porous MWCNT@NiMn-LDH composites based on ion transport of EDL coupled with ligand exchange mechanism. Separation and Purification Technology, 2019, 223: 154-161.

[19] LI M, LIU J, XU Y, et al. Phosphate adsorption on metal oxides and metal hydroxides: a comparative review. Environmental Reviews, 2016, 24(3): 319-332.

[20] PANG HONG-WEI, TANG HAO, WANG JIA-QI, et al. Ternary layered double hydroxide supported sulfide NZVI: efficient U(VI) elimination and mechanism. Journal of Inorganic Materials, 2020, 35(3): 381-389.

[21] SHAO M, LI Z, ZHANG R, et al. Hierarchical conducting polymer@clay core-shell arrays for flexible all-solid-state supercapacitor devices. Small, 2015, 11(29): 3530-3538.

[22] YU C, ZHANG L, SHI J, et al. A simple template-free strategy to synthesize nanoporous manganese and nickel oxides with narrow pore size distribution, and their electrochemical properties. Advanced Functional Materials, 2008, 18(10): 1544-1554.
[23] TASKOPRU T, BAYANSAL F, ŞAHIN B, et al. Structural and optical properties of Co-doped $\mathrm{NiO}$ films prepared by SILAR method. Philosophical Magazine, 2015, 95(1): 32-40.

[24] CHEN H, HU L, CHEN M, et al. Nickel-cobalt layered double hydroxide nanosheets for high-performance supercapacitor electrode materials. Advanced Functional Materials, 2014, 24(7): 934-942.

[25] MANIVASAKAN P, RAMASAMY P, KIM J. Use of urchin-like $\mathrm{Ni}_{(x)} \mathrm{Co}_{(3-x)} \mathrm{O}_{4}$ hierarchical nanostructures based on non-precious metals as bifunctional electrocatalysts for anion-exchange membrane alkaline alcohol fuel cells. Nanoscale, 2014, 6(16): 9665-9672.

[26] SUN B, HAO X, Wang Z, et al. Separation of low concentration of cesium ion from wastewater by electrochemically switched ion exchange method: experimental adsorption kinetics analysis. Journal of Hazardous Materials, 2012, 233: 177-183.

[27] CAI J, ZHANG Y, PAN B, et al. Efficient defluoridation of water using reusable nanocrystalline layered double hydroxides impregnated polystyrene anion exchanger. Water Research, 2016, 102: 109-116.

[28] GOH K, LIM T, BANAS A, et al. Sorption characteristics and mechanisms of oxyanions and oxyhalides having different molecular properties on $\mathrm{Mg} / \mathrm{Al}$ layered double hydroxide nanoparticles. Journal of Hazardous Materials, 2010, 179(1/2/3): 818-827.

[29] ZHANG G, LIU H, LIU R, et al. Removal of phosphate from water by a Fe-Mn binary oxide adsorbent. Journal of Colloid and Interface Science, 2009, 335(2): 168-174.

[30] YAN L, XU Y, YU H, et al. Adsorption of phosphate from aqueous solution by hydroxy-aluminum, hydroxy-iron and hydroxy-ironaluminum pillared bentonites. Journal of Hazardous Materials, 2010, 179(1/2/3): 244-250.

[31] DRENKOVA-TUHTAN A, MANDEL K, PAULUS A, et al. Phosphate recovery from wastewater using engineered superparamagnetic particles modified with layered double hydroxide ion exchangers. Water Research, 2013, 47(15): 5670-5677.

[32] SU Y, CUI H, LI Q, et al. Strong adsorption of phosphate by amorphous zirconium oxide nanoparticles. Water Research, 2013, 47(14): 5018-5026.

[33] QIU H, LIANG C, YU J, et al. Preferable phosphate sequestration by nano-La(III)(hydr)oxides modified wheat straw with excellent properties in regeneration. Chemical Engineering Journal, 2017, 315: $345-354$. 